\title{
Recomendación Clínica: Disponibilidad y Uso de Monitorización Perioperatoria
}

\author{
JOSÉ IGNACIO EGAÑA TOMIC. ${ }^{1}$, MD, PHD., PAULA FUENZALIDA SOLER. ${ }^{2}$, MD. CLAUDIA JIMÉNEZ \\ ESPERIDIÓN. ${ }^{2}$, MD. ÁLVARO JARA SCHNETTLER. ${ }^{3}$, FELIPE MALDONADO CANIULAO. ${ }^{2}$, MD, M.SC. \\ ANTONELLO PENNA SILVA. ${ }^{1}$, MD, PHD. WALDO MERINO URRUTIA ${ }^{4}$
}

\section{Lista de abreviaturas}

$\begin{array}{ll}\text { ASA } & \text { American Society of Anesthesiologists. } \\ \text { SACH } & \text { Sociedad Chilena de Anestesiología. } \\ \text { RCs } & \text { Recomendaciones Clínicas. } \\ \text { URPA } & \text { Unidad de Recuperación de Post Anestesia. } \\ \text { ECG } & \text { Electrocardiograma. } \\ \text { BNM } & \text { Bloqueo Neuromuscular. } \\ \mathrm{EtCO}_{2} & \begin{array}{l}\text { Concentración de } \mathrm{CO}_{2} \text { al final de la espira- } \\ \text { ción. }\end{array} \\ \text { TIVA } & \begin{array}{l}\text { Anestesia Total Intravenosa. } \\ \text { MINSAL }\end{array} \\ \text { MRADisterio de Salud, Chile. } \\ \end{array} \quad \begin{aligned} & \text { The Grading of Recommendations As- } \\ & \text { sessment, Development and Evaluation. }\end{aligned}$

\section{Definición de términos}

A. Anestesia general: Estado reversible de pérdida de conciencia inducida por fármacos.

B. Anestesia regional: Conjunto de variadas técnicas anestésicas que permiten la inducción de insensibilidad en una zona del cuerpo, de forma controlada y reversible.

C. Evaluación ASA: Sistema de clasificación que utiliza la Sociedad Americana de Anestesiólogos (ASA), para estimar estado fisiológico de los pacientes.
D. Evaluación preanestésica: Proceso de evaluación y estudio que precede a la administración de anestesia, tanto para procedimientos quirúrgicos como no quirúrgicos.

E. Perioperatorio: Corresponde al período que se extiende desde que se indica la realización o necesidad de cirugía, hasta el momento en que el paciente es dado de alta.

F. Sedación: Estado farmacológico de depresión variable del estado de conciencia, que proporciona comodidad y cooperación del paciente.

G. Unidad de Recuperación Postanestésicos (URPA): Sala destinada a proveer cuidados postanestésicos inmediatos de pacientes que han sido sometidos a cirugías o procedimientos diagnósticos o terapéuticos bajo anestesia, hasta que se alcancen criterios de alta predefinidos.

\section{Declaración de conflicto de interés de los par- ticipantes}

José Ignacio Egaña: no tiene conflictos de interés. Antonello Penna: no tiene conflictos de interés. Álvaro Jara: no tiene conflictos de interés. Felipe Maldonado: no tiene conflictos de interés. Jaime Escobar: no tiene conflictos de interés. Paula Fuenzalida: no tiene conflictos de interés.

Anestesiólogo, Profesor Asistente, Departamento de Anestesiología y Reanimación de la Universidad de Chile.

Anestesióloga, Instructor, Departamento de Anestesiología y Reanimación de la Universidad de Chile.

Anestesiólogo, Departamento de Anestesiología y Reanimación de la Universidad de Chile.

Anestesiólogo, profesor Asistente, Departamento de Cirugía, Traumatología y Anestesia Universidad de La Frontera.

Fecha de recepción: 27 de abril de 2018

Fecha de aceptación: 27 de abril de 2018

ORCID

https://orcid.org/0000-0002-4956-8444

Correspondencia:

Waldo Merino

Email: wamerino@gmail.com 
Claudia Jiménez: no tiene conflictos de interés.

Waldo Merino Urrutia: no tiene conflictos de interés.

\section{Desarrollo de la recomendación clínica}

\subsection{Introducción}

La anestesiología es una especialidad en constante cambio. En este contexto, la monitorización anestésica, entendida tanto para procedimientos bajo anestesia general, anestesia regional y cuidados anestésicos monitorizados, ha sido probablemente una de las áreas que más ha avanzado en su desarrollo en los últimos años. Con la intención de homogeneizar la práctica clínica profesional con estándares de calidad adecuados a los tiempos, señalando la mejor evidencia disponible relacionada a esta materia, la SACH ha considerado fundamental actualizar las últimas RCs publicadas el año 2003 con la finalidad de obtener un mejor desempeño clínico del profesional y que nuestros pacientes tengan un mayor beneficio de nuestro práctica. El objetivo global es brindar un marco académico y profesional acerca del uso de la monitorización en la práctica clínica anestésica, para optimizar el cuidado y seguridad de nuestros pacientes, considerando a la vez la distintas realidades en la que la anestesia es practicada en nuestro país. La guía está dirigida principalmente a los(as) anestesiólogos(as) y destaca en ella el rol preponderante de estos en el cuidado del paciente sometido a cualquier procedimiento anestésico. La presente guía no busca plantear una norma estricta sino recomendar conductas de buena práctica en relación a la evidencia actual, el criterio clínico y condición particular de cada paciente en específico, aspectos que determinarán finalmente la conducta adoptada por cada anestesiólogo(a) responsable.

\subsection{Objetivos}

La observación clínica y la evaluación por parte de un(a) anestesiólogo(a) son esenciales para la seguridad del paciente durante la anestesia. El objetivo de estas guías es, proporcionar las recomendaciones de monitorización perioperatoria para el seguimiento fisiológico de los pacientes, que de aplicarse en la gestión clínica cumplan con el fin de optimizar la seguridad del paciente y la calidad de la atención. Cada sección de estas directrices está sujeta a una revisión según la evolución de la tecnología y la práctica clínica.

\subsubsection{Escenarios clínicos a los que se refiere la $R C$}

\section{y condiciones de aplicación}

Las siguientes recomendaciones clínicas se aplican a todos los procedimientos que se realicen en pabellón bajo anestesia general, anestesia regional y cuidados anestésicos monitorizados. Así como también a los procedimientos anestésicos realizados fuera de pabellón y a la monitorización en las URPAs. Es importante señalar que las RCs son una exhaustiva revisión de expertos, donde se puede reconocer algunas condiciones de equipamiento e infraestructura que son necesarias para cumplir un desempeño profesional acorde a lex artis. Estas condiciones, en general, son una responsabilidad exclusiva y excluyente del administrador o gestor de los recursos en el sector público y privado. Por esta razón la SACH recomienda a sus asociados hacer presentaciones escritas para que los administradores y gestores se mantengan razonablemente informados en estas materias. Las comunicaciones respectivas se pueden canalizar a través de la $\mathrm{SACH}$, ello permite conducir y facilitar los procesos en las instituciones sanitarias que sostengan condiciones de trabajo y clínicas deficitarias[2].

\subsubsection{Eventuales situaciones en las que no es aplicable la RC}

Se excluye del interés de ésta RC la monitorización en el postoperatorio tardío, entendiéndose como tal el período de tiempo que transcurre una vez que el paciente abandona la URPA y la monitorización durante el traslado de pacientes. En los procedimientos fuera de pabellón, esta recomendación hace alusión solamente a la monitorización, dado que otros aspectos de este tema se tratan extensamente en la recomendación clínica "Anestesia Fuera de Pabellón" de la Sociedad de Anestesiología de Chile[3].

\subsection{Métodos}

\subsubsection{Identificación de documentos fuentes. Sín-} tesis de evidencia

\subsubsection{Identificación de prestaciones vinculadas al problema de salud}

Se realizó una búsqueda dirigida de los artículos atingentes al tema publicados en los últimos 10 años en la base de datos de MEDLINE, utilizando su motor de búsqueda PubMed. Las palabras claves utilizadas para la búsqueda fueron: (Guideline OR Guidelines OR Recommendation OR Standards) AND Monitoring AND (anesthesia OR intraoperative), con lo cual obtuvimos un total de 1.447 artículos. Posteriormente, tres anestesiólogos conocedores del tema (AJ, AP, JE) seleccionaron de manera independiente los artícu- 
los atingentes con la búsqueda. Los artículos seleccionados se determinaron si los tres anestesiólogos estuvieron de acuerdo. En total se seleccionaron 8 artículos, de los cuales 3 fueron guías[4-6] y 5 comentarios o editoriales[7-11] acerca de éstas. Finalmente, se decidió realizar una búsqueda dirigida de las recomendaciones de las Sociedades Anestésicas y de Salud relevantes a nivel mundial: American Society of Anesthesiologists (ASA)[12], Australian and New Zealand College of Anaesthetists (ANZCA)[13], World Health Organization (WHO)[14], y además se seleccionó la recomendación previa de nuestra Sociedad[15]. En resumen, estas recomendaciones se basan en lo expuesto por los 12 artículos seleccionados.

Además, con respecto a los temas de monitorización del bloqueo neuromuscular, monitorización de la profundidad anestésica y analizador de gases, se obtuvo los artículos originales que dan sustento a la evidencia clínica mencionada en las guías clínicas antes mencionadas.

\subsubsection{Efectividad de las intervenciones}

La mayoría de los temas que son expuestos en esta RC son considerados como "Estándares mínimos de atención e implican un deber ético intrínseco a la práctica médica", por lo que son intervenciones en salud sobre las cuales no existen incertidumbres sobre la pertinencia de su aplicación o respecto a la efectividad y seguridad de las mismas. Por lo que se han difundido ampliamente a nivel mundial y su efectividad es citada en la literatura como un hecho suficientemente demostrado. La calidad de la evidencia y la fuerza de la recomendación se describe en los temas en que se estimó pertinente.

\subsubsection{Efectos adversos de las intervenciones}

La monitorización estándar en sí misma no genera efectos adversos por lo que no es un tema relevante en estas recomendaciones. Sin embargo, en la instalación de dispositivos de monitorización invasiva se puede observar la aparición de eventos adversos inherentes a cada uno de ellos. La decisión de la instalación de la monitorización invasiva debe suponer un mayor beneficio al paciente considerando un eventual riesgo de una complicación, local/sistémica, en la instalación o por presencia prolongada en el tiempo del dispositivo de monitorización invasiva.

Los resultados de un accidente anestésico completamente prevenible como consecuencia de una monitorización inadecuada o insuficiente pueden ser catastróficos. El hecho de que los riesgos asociados a la monitorización en sí son extremadamente bajos sostienen la recomendación necesaria de su utilización.

\subsubsection{Condiciones de calidad de las interven- ciones}

Las intervenciones que se discuten en esta RC, como se señaló previamente, son considerados por este equipo de trabajo como "Estándares mínimos de atención e implican un deber ético intrínseco a la práctica médica" y han sido ampliamente presentados y recomendados en guías de práctica clínica o recomendaciones clínicas por otras sociedades y agencias gubernamentales a nivel mundial. Son intervenciones en salud, sobre las cuales no existen incertidumbres respecto a la efectividad y seguridad de las mismas o sobre la pertinencia de su aplicación.

\subsubsection{Métodos de consenso utilizados para la formulación de recomendaciones}

Están establecidos en el documento "Orientaciones para la Elaboración y Actualización de Recomendaciones Clínicas de la Sociedad de Anestesiología de Chile".

\subsubsection{Grados de evidencia y niveles de recomen- dación utilizados en la RC}

Para muchas de las recomendaciones no existe evidencia proveniente de ensayos controlados. En general, las acciones o dispositivos aquí señalados son estándares avalados por la comunidad de anestesiológica mundial, en su búsqueda de la seguridad del paciente.

Es así que muchas de las guías clínicas de sociedades anestesiológicas han adquiridos estándares sin un nivel de evidencia explícito. Esto porque, como se mencionó anteriormente, son intervenciones en salud sobre las cuales no ha existido incertidumbres respecto a la efectividad y seguridad de las mismas, o sobre la pertinencia de su aplicación. Aún así, en la elaboración de esta RC se efectuó la necesaria actualización de la evidencia disponible a través de herramientas metodológicas actualizadas, asumiendo el permanente cambio tecnológico y desarrollo de la especialidad. De acuerdo con los lineamientos entregados por la $\mathrm{SACH}$ a través del documento "Orientaciones para la Elaboración y Actualización de Recomendaciones Clínicas de la Sociedad de Anestesiología de Chile".

Así, para la evaluación y redacción de las recomendaciones se utilizó el sistema GRADE recomendado por el MINSAL. Sin embargo, es necesario mencionar lo establecido por el MINSAL que "... no todas las preguntas relevantes para una guía de práctica clínica pueden, ni deben, responderse con evidencia científica. Cuando las características de una intervención hacen que no sea posible o éticamente válido realizar estudios respecto de su efectividad, y se considera en 
consenso que dicha intervención refleja un estándar mínimo de atención, el panel puede emitir una recomendación de buena práctica"[1]. Esto se presenta especialmente en la siguiente recomendación clínica en la que las publicaciones, y este grupo de trabajo en particular, señalan estándares mínimos en monitorización, avalados por la comunidad de anestesiológica mundial, aún con evidencia insuficiente, pero de incuestionable pertinencia y aplicabilidad.

\section{Recomendaciones}

\subsection{Generalidades}

En estas recomendaciones se entenderá por monitorización al proceso que implica la observación y el chequeo continuo del progreso y la calidad, en un periodo de tiempo, de la condición clínica de un paciente. Ésto incluye la observación clínica y las mediciones fisiológicas relevantes, como también, al registro de ellas durante el periodo de tiempo que dure la monitorización y, por ello, al tiempo que dura la responsabilidad clínica[13].

El monitoreo de las variables fisiológicas provee información y una retroalimentación de la respuesta del organismo a las intervenciones y a los cambios de la condición clínica. Esto permite una mayor precisión en el manejo para alcanzar mejores desenlaces y disminuir al mínimo las complicaciones[13].

\subsubsection{Presencia de un anestesiólogo(a) en pabe- Ilón}

Este grupo de trabajo considera en consenso que dicha intervención refleja un estándar mínimo de atención y un deber ético intrínseco a la práctica médica. Por lo anterior, recomienda fuertemente la presencia de un anestesiólogo por y en pabellón.

Comentario: La observación y el análisis continuo de la monitorización del paciente debe ser realizada durante todo el periodo de una anestesia general, regional, o a un cuidado anestésico monitorizado por el anestesiólogo(a) tratante[5, 13]. Este anestesiólogo(a) debe estar asignado a un único pabellón y responsabilizarse por el cuidado del paciente. El anestesiólogo(a) tratante, eventualmente, puede delegar el cuidado del paciente a otro anestesiólogo(a), a un residente de anestesiología o a un enfermero(a) o a un técnico(a) de anestesia[6]. Al delegar el cuidado del paciente a un residente de anestesiología o a un enfermero(a) o a un técnico(a) de anestesia, el anestesiólogo(a) tratante sigue siendo el responsable del manejo anestésico del paciente[6]. Cuando se delega a un resi- dente de anestesiología o a un enfermero(a) o a un técnico(a) de anestesia, el anestesiólogo(a) tratante debe permanecer disponible de manera inmediata[6].

\subsubsection{Monitorización del paciente}

El objetivo de la monitorización es observar y registrar la evolución temporal de las variables fisiológicas básicas durante una intervención que requiera de una anestesia general, regional o de cuidados monitorizados anestésicos. Los equipos utilizados en una monitorización deben ser revisados previo al inicio de cada caso según los chequeos de seguridad establecidos.

La SACH recomienda como monitorización la evaluación de la oxigenación, ventilación, circulación, temperatura, además de la monitorización del bloqueo neuromuscular, de la profundidad anestésica y de la concentraciones del agente inhalatorio según corresponda al caso clínico. Estas recomendaciones corresponden a una actualización de las recomendaciones de la SACH publicadas el año 2003[15].

\subsubsection{Oxigenación}

Este grupo de trabajo considera en consenso que la monitorización de la oxigenación es un estándar mínimo de atención y un deber ético intrínseco a la práctica médica. Por ello, recomendamos fuertemente:

Evaluación visual del paciente: Durante todo procedimiento se recomienda el acceso al paciente para poder realizar una observación clínica de su perfusión tisular manteniendo una adecuada iluminación. Excepcionalmente, para ciertas cirugías, la falta del acceso al paciente puede permitirse teniendo un plan de aproximación en caso de necesidad.

Uso de Oximetría de pulso: Se recomienda la medición de oxigenación de la hemoglobina en todo procedimiento anestésico mediante la utilización de un oxímetro de pulso que entregue la onda de pulso para confirmar la presencia de la onda de latido. Además, se debe utilizar la señal audible del latido con cambios de tono de la señal como alarma adicional de la caída de la oxigenación de la hemoglobina. Se recomienda el uso y la configuración de las alarmas de hipoxemia.

Uso de Oximetría en línea: Se recomienda el uso de un sensor de oxígeno continuo en la máquina de anestesia en todo paciente sometido a una anestesia con un sistema de ventilación. Este sistema permite monitorizar la concentración de oxígeno inspirado y espirado de manera continua. Se recomienda la configuración de alarmas que indiquen el límite inferior de oxigenación. 
Uso de Alarmas: Previo a cada procedimiento anestésico y en cada paciente se recomienda revisar y ajustar las alarmas del oxímetro de pulso y del oxímetro en línea configurando el límite inferior al inicio de todo procedimiento anestésico. Estas alarmas deben tener una señal audible y una señal luminosa.

\subsubsection{Ventilación}

Este grupo de trabajo considera en consenso que la monitorización de la ventilación es un estándar mínimo de atención y un deber ético intrínseco a la práctica médica. Por ello, recomendamos fuertemente:

Evaluación visual del paciente: Permite obtener datos de la mecánica ventilatoria. Pese a que la visualización de la excursión torácica durante el movimiento de la bolsa reservorio pueden ser útiles para evaluar la ventilación, no son suficientes para la confirmación de las variables ventilatorias más específicas. Se recomienda el uso de la auscultación para evaluar la ventilación de ambos campos pulmonares.

Comprobación de la intubación: se recomienda el uso de la concentración de $\mathrm{CO}_{2}$ al final de la espiración $\left(\mathrm{EtCO}_{2}\right)$ y la onda de capnografía para la confirmación de la intubación endotraqueal y la correcta instalación de los dispositivos supraglóticos. Los signos clínicos tienen una utilidad secundaria en la comprobación de la intubación endotraqueal. Se debe revisar el estado del testigo del tubo endotraqueal (Cuff) y el estado de insuflación de dispositivos supraglóticos.

Capnografía: Se recomienda en todo paciente bajo anestesia general o sedación moderada y profunda el monitoreo contínuo del $\mathrm{CO}_{2}$ en la inspiración y espiración, siendo relevante tanto la presencia como el valor de la $\mathrm{EtCO}_{2}$. Esta monitorización debe estar presente desde la inducción hasta la extubación, retiro del dispositivo supraglótico o tras la superficialización de la sedación. Además, es importante destacar que no sólo permite la evaluación de la ventilación, sino que también aporta información sobre el gasto cardiaco.

Medición de presión de vía aérea: Se recomienda la monitorización de la presión de vía aérea en todo paciente bajo anestesia general.

Alarmas: previo a cada procedimiento anestésico y en cada paciente, se deben revisar y ajustar las alarmas de la capnografía y de la presión de vía aérea, configurando los límites inferiores y superiores de las mismas. Estas deben contar con una señal audible y una señal luminosa.

\subsubsection{3.- Circulación}

Este grupo de trabajo considera en consenso que la monitorización de la circulación es un estándar mínimo de atención y un deber ético intrínseco a la práctica médica. Por ello, recomendamos fuertemente monitorizar:

Electrocardiograma: Se recomienda el uso de monitorización continua de la onda electrocardiográfica para todo paciente bajo una anestesia general, regional, sedación o un cuidado anestésico monitorizado. El análisis de segmento ST se aconseja para todo paciente.

Presión arterial: Se recomienda la monitorización intermitente de la presión arterial en todo paciente bajo una anestesia general, regional, sedación o un cuidado anestésico monitorizado. El intervalo de medición no debe ser mayor a los 5 minutos. Debe existir mangos de presión adecuados para el tamaño de cada paciente. Esta recomendación pone especial énfasis en grupos especiales de pacientes como pediátricos y obesos. La medición invasiva y continua de la presión arterial debe estar disponible para todo paciente de ser necesario.

\subsubsection{Temperatura}

Este grupo de trabajo considera en consenso que la monitorización de la temperatura es un estándar mínimo de atención y un deber ético intrínseco a la práctica médica. Por ello, recomendamos fuertemente el uso de termómetro para todo paciente que ingresa a procedimientos de más de 30 minutos de duración. La medición central de la temperatura debe estar disponible para todo paciente.

\subsubsection{Monitorización del bloqueo neuromus- cular}

Recomendamos que la monitorización de la función neuromuscular esté disponible para todos los pacientes en quienes se ha inducido el bloqueo neuromuscular (BNM) siendo deseable que la evaluación se realice a lo largo de todo el proceso anestésico y, particularmente, para comprobar la reversión adecuada de dicho bloqueo, previo a la extubación del paciente en función de evitar el bloqueo neuromuscular residual[ $[5,13]$.

Recomendamos el uso de estimuladores de nervio periférico cuantitativos ya que permiten de mejor manera garantizar el retorno de una función motora adecuada Esta se obtiene cuando la relación en el protocolo "tren de cuatro" (TOF, por sus siglas en inglés) es mayor a 0.9 o $90 \%[5,13]$. El uso de fármacos antagonistas de bloqueo neuromuscular debe ser guiado por monitorización de bloqueo neuromuscular.

Este grupo recomienda fuertemente el uso de 
estimuladores de nervio periférico cuantitativos para evitar el BNM residual en el posoperatorio[20-23].

Calidad de la evidencia es moderada. Recomendación Fuerte.

\subsubsection{Monitorización de profundidad anesté- sica.}

La utilización de los monitores de profundidad anestésica han sido recomendados cuando hay un riesgo alto de despertar intraoperatorio (awareness) o en pacientes que pueden tener efectos adversos por una profundidad anestésica aumentada o en los pacientes a los cuales se le suministra una anestesia total intravenosa (TIVA, por sus siglas en inglés). Sin embargo, la eficacia de estos monitores para predecir los episodios de awareness accidental o el nivel anestésico aún son inconsistentes y están en debate. Pero estos monitores entregan datos adicionales para las decisiones clínicas. Por ello, se sugiere utilizar monitores de profundidad anestésica en pacientes con bloqueo neuromuscular bajo TIVA[24], pacientes con un riesgo alto de awareness[24] y en adultos mayores con riesgo de cursar con cuadros de delirium postoperatorio[25]. La monitorización debe comenzar con la inducción y finalizar con la emergencia anestésica[5].

Este grupo de trabajo considera que el uso de monitores de profundidad anestésica para la prevención de awareness no es recomendado al utilizar hipnóticos inhalatorios con analizador de gases.

Calidad de la evidencia alta. Recomendación Fuerte.

El uso de monitores de profundidad anestésica está recomendado fuertemente al utilizar TIVA y BNM a pesar de que el nivel de evidencia es bajo.

Calidad de la evidencia baja. Recomendación Fuerte.

Se recomienda el uso de estos monitores para la prevención de delirium postoperatorio en adultos mayores.

Calidad de la evidencia es moderada. Recomendación débil.

\subsubsection{Analizador de gases.}

Este grupo de trabajo recomienda el uso de analizador de gases, cuya finalidad es identificar el agente y determinar su concentración parcial durante la inspiración y al final de la espiración (Et, por sus siglas en inglés). El uso del analizador de gases es esencial en toda anestesia general en que se utilice un agente anestésico inhalatorio u óxido nitroso[5,13,24].

Recomendamos que las alarma sean ajustadas a un valor mínimo adecuado y efectivo para estimar la profundidad anestésica[5].
Este grupo recomienda fuertemente la monitorización continua de la concentración del agente inhalatorio para titular una dosis adecuada del hipnótico, pese a que la calidad de la evidencia es moderada.

Calidad de la evidencia es moderada. Recomendación Fuerte.

\subsubsection{Otros monitores}

Cuando la condición clínica del paciente lo indique, deben estar disponibles los equipos para monitorizar otras variables fisiológicas como, por ejemplo, presiones intravasculares, el gasto cardiaco o variables bioquímicas y hematológicas. El uso de monitoreo adicional queda a discreción del anestesiólogo(a)[5,13].

\subsubsection{Monitorización en la unidad de recupera- ción anestésica}

Todo centro donde se realicen intervenciones que requieran de una anestesia general, regional o de cuidados monitorizados anestésicos debe tener una unidad de recuperación anestésica (URPA). El anestesiólogo(a) debe acompañar al paciente a la URPA, comunicar la información necesaria y escribir las indicaciones apropiadas. Si la condición clínica del paciente y la distancia desde el pabellón a la URPA lo indica, el transporte del paciente debe ser monitorizado y con oxígeno suplementario.

Debe haber un anestesiólogo(a) asignado a la URPA, quien es el responsable de los cuidados anestésicos en la unidad de recuperación. El cuidado del paciente no debe ser delegado al personal de enfermería de la URPA hasta que el anestesiólogo(a) esté seguro que el paciente puede ser observado y cuidado por dicho personal. El alta desde la URPA es responsabilidad del anestesiólogo(a) tratante o el anestesiólogo(a) asignado a los cuidados en dicha unidad[6].

En toda URPA debe haber un equipo de emergencia y de resucitación. Cada paciente debe tener disponible oxígeno suplementario, un sistema de aspiración, y un sistema de registro de los signos vitales y de las observaciones e incidentes. El monitoreo utilizado en la URPA debe ser apropiado para el estado del paciente y debe estar disponible una gama completa de dispositivos de monitoreo. La monitorización debe mantenerse hasta que el paciente logre recuperarse completamente de los efectos de la anestesia. El estar "completamente recuperado" significa que no requiera algún apoyo en la vía aérea, mantenga una ventilación espontánea, esté alerta, responda a las órdenes y se comunique, de acuerdo a los test de recuperación postanestésica como, por ejemplo, Aldrete. Entonces, el monitoreo debe mantenerse para detectar rápidamente alteraciones en la vía aérea, 
ventilación y circulación[5]. En algunas circunstancias, puede ser considerado aceptable transferir al paciente directamente a otra unidad de cuidados o evitar la URPA, si la otra unidad posee un nivel de cuidado apropiado a la condición del paciente y bajo el criterio del anestesiólogo(a) tratante, por ejemplo Unidad de Cuidados Críticos.

En resumen, este grupo considera en consenso que la monitorización en la URPA es un estándar mínimo de atención y un deber ético intrínseco a la práctica médica. Por ello, recomendamos fuertemente utilizar[5]: oxímetro de pulso, presión arterial no invasiva, electrocardiograma, temperatura y capnografía, si el paciente tiene un tubo endotraqueal o un dispositivo supraglótico o está profundamente sedado.

\subsubsection{Monitorización en procedimientos fuera de pabellón}

Cuando los anestesiólogos(as) son llamados a administrar una anestesia general, regional o a un cuidado anestésico monitorizado fuera de pabellón se deben aplicar las mismas normas mínimas de monitoreo ya explicitadas en este documento[5].

Para una información más detallada de los procedimientos fuera de pabellón, puede revisar las recomendaciones de la SACHILE de Anestesia Fuera de Pabellón[3].

\section{Herramientas de ayuda para la decisión. Ma- terial de apoyo}

Ver sección 1.3. Síntesis de recomendaciones.

\section{Repercusiones económicas potencialmente re- levantes de la aplicación de la RC}

Estas recomendaciones clínicas potencialmente tienen un impacto económico en los servicios de pabellón y anestesia debido a que podrían generar la necesidad de adquirir aquellos monitores que no han tenido un uso extendido, tales como los estimuladores de nervio periférico cuantitativos para objetivar el bloqueo neuromuscular, los monitores de la profundidad anestésica, entre otros. Pese a este mayor costo, el que puede generar una demora en la implementación de las recomendaciones clínicas, creemos relevante que los servicios clínicos que realizan procedimientos bajo una anestesia general, regional o de cuidados monitorizados, tienen una URPA o realizan procedimientos fuera de pabellón, deben plantearse como objetivo la adquisición de los equipos descritos en esta recomendación dado que va en directo beneficio con el resultado clínico de los pacientes.

\section{Referencias}

1. Borghero Lasagna Francesca BPR, Carrasco Labra Alonso, Dembowski Sandoval Natalia, Kraemer Gómez Patricia, Mendoza Van der Molen Carolina, Neumann Burotto Ignacio, Pantoja Calderón Tomás, Rada Giacaman Gabriel, Rodríguez Camus María Francisca, Tohá Torm María Dolores. Manual Metodológico: Desarrollo de Guías Práctica Clínica. 2014.

2. SACHILE. Orientaciones para la elaboración y actualización de Recomendaciones Clínicas de la Sociedad de Anestesiología de Chile www.sachile.cl2018

3. SACHILE. Recomendaciones de la Sociedad de Anestesiología de Chile para la Anestesia fuera de
Pabellón https://www.sachile.cl/ view/pdf_pages/anestesia_fuera_pabellon.pdf2011

4. Alvarez Gómez JA, Arino Irujo JJ, Errando Oyonarte $\mathrm{CL}$, Matínez Torrente F, Roige i Sole J, Gilsanz Rodríguez F, et al. Use of neuromuscular blocking agents and reversal of blockade: guidelines from Sociedad Espanola de Anestesiologia, Reanimacion y Terapeutica del Dolor. Rev Esp Anestesiol Reanim. 2009;56(10):616-27.

5. Checketts MR, Alladi R, Ferguson K, Gemmell L, Handy JM, Klein $A A$, et al. Recommendations for standards of monitoring during anaesthesia and recovery 2015: Association of Anaesthetists of Great Britain and Ireland. Anaesthesia. 2016;71(1):85-93.
6. Merchant $R$, Chartrand D, Dain S, Dobson G, Kurrek MM, Lagace $A$, et al. Guidelines to the Practice of Anesthesia - Revised Edition 2016. Can J Anaesth. 2016;63(1):86-112.

7. Adekanye $O$, Dugani $S$, Wilkes AR, Srinivas K, Hodzovic I. AAGBI guidelines on the use of neuromuscular blockade monitoring. Anaesthesia. 2009;64(8):923-4.

8. Checketts MR. AAGBI recommendations for standards of monitoring during anaesthesia and recovery 2015. Anaesthesia. 2016;71(4):470-1.

9. Dalton AJ, Millar F. Neuromuscular monitoring and the AAGBI 2016 monitoring guidelines. Anaesthesia. 2016;71(8):981-2.

10. Lumb AB, McLure HA. AAGBI recommendations for standards 
of monitoring during anaesthesia and recovery 2015 - a further example of 'aggregation of marginal gains'. Anaesthesia. 2016;71(1):3-6.

11. Checketts MR, Jenkins $B$, Pandit JJ. Implications of the 2015 AAGBI recommendations for standards of monitoring during anaesthesia and recovery. Anaesthesia. 2017;72 Suppl 1:3-6.

12. STANDARDS FOR BASIC ANESTHETIC MONITORING. American Society of Anesthesiologists. 2015.

13. Anaesthetists AaNZCo. Guidelines on Monitoring During Anaesthesia. ANZCA DOCUMENTS. 2015.

14. WHO. WHO Guidelines for Safe Surgery 2009. Safe Surgery Saves Lives. 2009. In: WHO Library.

15. Disponibilidad y Uso de Monitorización Intraoperatoria. Revista Chilena de Anestesia. 2003:16.

16. Neumann I, Pantoja T, Penaloza B, Cifuentes L, Rada G. [The GRADE system: a change in the way of assessing the quality of evidence and the strength of recommendations]. Rev Med Chile 2014;142(5):630-5.
17. Kristensen SD, Knuuti J, Saraste A, Anker S, Botker HE, De Hert S, et al. 2014 ESC/ESA Guidelines on non-cardiac surgery: cardiovascular assessment and management: The Joint Task Force on non-cardiac surgery: cardiovascular assessment and management of the European Society of Cardiology (ESC) and the European Society of Anaesthesiology (ESA). Eur J Anaesthesiol. 2014;31(10):51773.

18. Recommendations for guidelines productions. A document for task forces members responsible for the production un updating of ESC Guidelines. Committe for Practice Guidelines of the European Society of Cardiology. https://www.escardio.org/ static_file/Escardio/Guidelines/ about/ESC_Guidelines_for_Guidelines_Update_2012_for_web. pdf2012 [

19. Ricardo Bustamante. Editorial: Recomendaciones Clínicas. Rev Chil Anest. 2012;41:3.

20. Bulka CM, Terekhov MA, Martin BJ, Dmochowski RR, Hayes RM, Ehrenfeld JM. Nondepolarizing Neuromuscular Blocking Agents, Reversal, and Risk of Postopera- tive Pneumonia. Anesthesiology. 2016;125(4):647-55.

21. Fortier LP, McKeen $D$, Turner $K$, de Medicis $E$, Warriner $B$, Jones PM, et al. The RECITE Study: A Canadian Prospective, Multicenter Study of the Incidence and Severity of Residual Neuromuscular Blockade. Anesth Analg. 2015;121(2):366-72.

22. Murphy GS, Brull SJ. Residual neuromuscular block: lessons unlearned. Part I: definitions, incidence, and adverse physiologic effects of residual neuromuscular block. Anesth Analg. 2010;111(1):120-8.

23. Naguib M, Brull SJ, Johnson KB. Conceptual and technical insights into the basis of neuromuscular monitoring. Anaesthesia. 2017;72 Suppl 1:16-37.

24. Avidan MS, Mashour GA. Prevention of intraoperative awareness with explicit recall: making sense of the evidence. Anesthesiology. 2013;118(2):449-56.

25. Aldecoa C, Bettelli G, Bilotta F, Sanders RD, Audisio R, Borozdina $A$, et al. European Society of Anaesthesiology evidence-based and consensus-based guideline on postoperative delirium. Eur J Anaesthesiol. 2017;34(4):192-214. 